\title{
Subjective contours independent of subjective brightness
}

\author{
COLIN WARE \\ Scarborough College, University of Toronto, Ontario MIC IA4, Canada
}

\begin{abstract}
Two theories of subjective contours are distinguished according to the interrelationship of subjective contours and subjective brightness effects. In one view, subjective contours are illusory brightness gradients generated from grouped local brightness effects. In another view, subjective contours are the edges of subjective forms created on the basis of gestalt factors; subjective brightness is a secondary consequence of form perception. Two experiments which use rating scales to separate judgments of subjective contour and subjective brightness are presented. The first shows that subjects may judge contour to be strong when there is no subjective brightness gradient. In the second, gestalt grouping factors are shown to be more important than factors which should influence brightness according to local effects theories. Both experiments support the view that subjective brightness occurs through interactions at the level of form perception.
\end{abstract}

Subjective contours are illusory edges perceived in regions that are physically homogeneous. These edges are sometimes perceived as like the edges of illusory objects (Kanizsa, 1955) and sometimes they seem to be brightness edges (Kennedy, 1979). This paper concerns the interaction between the impression of "edgeness" and the impression of brightness. On this basis, two kinds of theories can be distinguished."

According to the first, whose main exponents are Bradley and Dumais (1975) and Kanizsa $(1975,1976)$, gestalt factors result in the creation of a subjective form which subsequently contrasts with surrounding regions to seem brighter. ${ }^{2}$ This kind of theory can be called a "gestalt-contingent" theory.

According to the second kind of theory, subjective contours and subjective brightness are the result of local brightening and darkening effects. Thus, the ends of lines are held to induce local contrast effects (Day \& Jory, 1978; Frisby \& Clatworthy, 1975; Kennedy \& Lee, 1976), and local assimilation may occur between pattern elements (Day \& Jory, 1978; Kennedy, 1979). The theories of Day and Jory, Frisby and Clatworthy, and Kennedy and Lee differ in aspects of the specific local effects postulated. However, they are similar in that they all include local brightening at the ends of lines, and they include the idea that grouping (either perceptual or physical) of these local effects results in a region that is brighter or darker than surrounding regions. The brightness gradient at the boundary of such a region is the subjective contour. This kind of theory can conveniently be called a "local-effects" theory for its emphasis on local special brightness effects.

The author's present address: Division of Physics, National Research Council, Ottawa, Ontario K1A OR6, Canada.
One clear difference between the two types of theory is that, according to local effects theories, subjective contours are brightness gradients and so judgments of contour strength and subjective brightness should be interrelated in the following two ways: When there is no brightness, there should be no subjective contour, and judged brightness and judged contour should be positively correlated across displays with a gradient value close to one. Conversely, according to gestalt-contingent theories subjective contour may be largely independent of subjective brightness, since the gestalt factors which induce subjective form may be present while the contrast factors may be absent. For example, the subjective contour might be the perceived depth edge at the boundary of a form, or like a crack where the subjective form abuts adjacent forms (Kennedy, 1975). In either case, no brightness gradient is necessary for there to be contour perception.

A study by Jory and Day (1979) provided support for local effects theories by showing that versions of patterns isoluminant with, though a different color from, their backgrounds gave reduced ratings of contour and brightness compared with versions that differed in brightness from their backgrounds. The form constraints were held constant through this manipulation while contrast factors were altered. However, Gregory (1977) has argued that isoluminance may be a condition which also weakens form perception. If this were the case, gestalt-contingent theories would also predict reduced contour and brightness, since the gestalt would be weakened by isoluminance.

The purpose of this presently reported research was to further assess the interrelationship of subjective brightness and subjective contour. 


\section{EXPERIMENT 1}

The idea behind the first experiment was to hold constant a gestalt factor which might be expected to induce subjective contours and to vary a factor expected to alter the subjective brightness relationship between two regions of the display (according to gestalt-contingent and local-effects theories). A set of displays was produced, with each display divided into three regions, a central horizontal strip flanked above and below by wider strips. By varying the relative densities of parallel vertical lines within the strips, subjective brightness between the lines could be manipulated. That is, according to local effects theories, when there are more lines in flanking regions, more line end brightnesses will be concentrated in the central region and more darkening effects will occur in the flanking regions. Hence the central region will seem brighter than flanking regions. Conversely, when there is a greater density in the central region, the induced brightnesses will be reversed and the flanking regions will seem brighter. At some point in a series of displays, a point should be reached at which brightening and darkening effects are balanced across the three regions. At this point-according to local effects theories-the impression of a subjective contour should disappear.

While relative line densities are varied, by holding constant the number of lines terminating at the boundaries of the central strip, a gestalt factor which might be expected to be important in influencing contour strength could be held constant. Illustrations of three displays made according to these constraints are given in Figure 1.

(a)

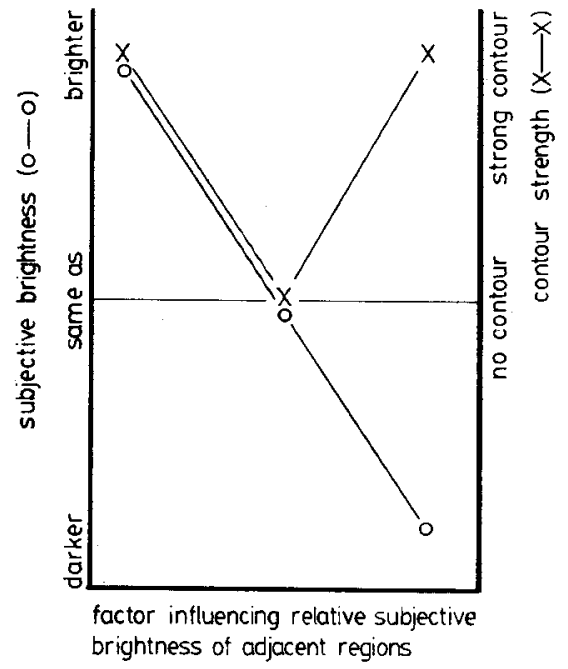

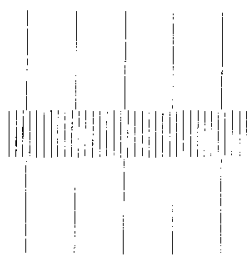
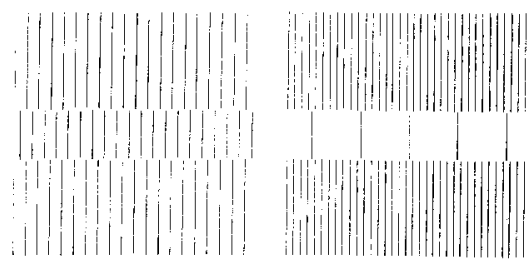

Figure 1. Three of a sequence of stimulus patterns wherein the number of lines terminating along the central boundary is held constant while line density in central and flanking regions varies. Subjective contours can be seen separating the regions.

The predictions of the two types of theory are graphed in Figure 2. According to local effects theories, the impression of contour should be directly related to the impression of a brightness difference between central and flanking regions. As the impression of a brighter central region is reduced, contour strength should likewise be diminished until the central and flanking regions seem the same and there is no subjective contour. As the subjective brightness of the central region is reduced still further, subjective contour should increase with the increasing brightness difference. This interaction is diagramed in Figure 2(a).

According to gestalt-contingent theories, the subjective brightness should vary in the same way as the relative line densities change; when flanking regions contain greater line density, the central region should seem brighter, and vice versa. However, the impression of a subjective boundary should remain strong throughout the sequence of displays, since the number of line terminations remains constant. This relation is graphed in Figure 2(b).

(b)

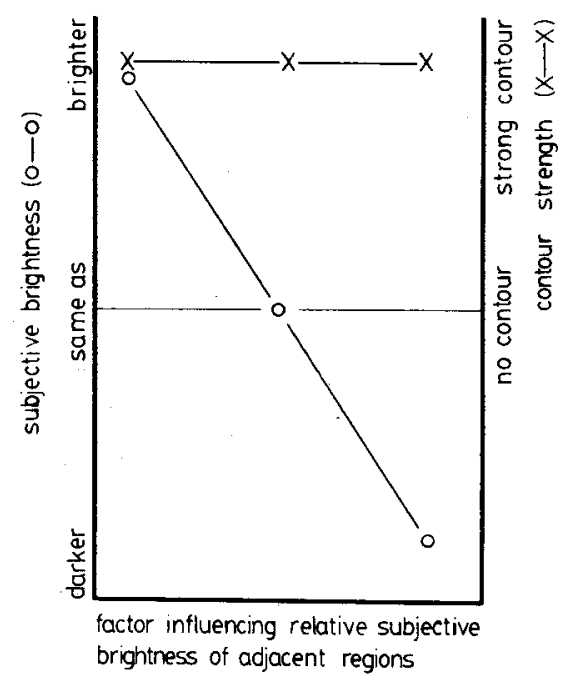

Figure 2. Two hypothetical graphs showing predicted interrelationship of subjective contour and subjective brightness according to two theories of subjective contours. (a) The prediction of local effects theories in which subjective contours are brightness gradients. (b) The prediction of gestaltcontingent theories in which contour is independent of brightness. 


\section{Method}

Subjects. The subjects were 14 individuals between the ages of 18 and 30 . Each had 20/20 vision or better (corrected in some cases), as measured by a Schnellen eye chart. About half the subjects were students; the other half were from a variety of occupations.

Stimuli and Apparatus. The stimuli consisted of seven $10-\mathrm{cm}$ squares of illustration board, each divided into three regions, a $2-\mathrm{cm}$ central strip flanked by two $4-\mathrm{cm}$ strips. Each region contained a number of vertical ruled lines $.04 \mathrm{~cm}$ wide. The ratios of line densities in inner and flanking regions were: 1:7, 2:6, 3:5, 4:4, $5: 3,6: 2,7: 1$. At the viewing distance of $114 \mathrm{~cm}$, the numbers given in the above ratios correspond to lines per degree of visual angle. Two standard, household $60-\mathrm{W}$ tungsten light bulbs were arranged so as to cast light on the displays from a distance of $42 \mathrm{~cm}$ at an angle of $45 \mathrm{deg}$ from either side. The illumination so obtained was found to be approximately $60 \mathrm{fc}$ as measured by a Macbeth illuminometer.

Procedure. The subjects were first given a training program, which consisted of an illustrated test describing some well-known subjective contour effects. For example, Kanizsa's (1955) triangle pattern was reproduced, and the subjects were instructed that an illusory bright overlaying triangle was a common subjective impression obtained from this display. The subjects were also instructed in the use of two rating scales. A 6-point scale (0 through 5) was used for rating subjective contour, with 0 representing no contour and 5 representing strong contour. Subjective contour was defined as "the impression that a line or edge exists in the region indicated. This may appear as like a thin wire, a crack, the edge of a shape, or the edge of a bright region. The second rating scale had 11 points, $(-5$ through +5 , with -5 representing a darker region, 0 representing no subjective brightness difference, and +5 representing a brighter region. Subjective brightness was defined as the impression that the spaces between the inducing lines seemed "brighter, lighter, or darker" in the central strip than did line interspaces in surrounding regions. The displays were presented to each subject twice, once in a sequence starting with the widest spacing in the central region and once in the reverse sequence. Half the subjects received one sequence first, and the other half received the other sequence first. ${ }^{3}$ Subjects were asked to rate the subjective boundary of the central strip and the subjective brightness of the spaces between the lines in the central strip.

\section{Results and Discussion}

The results are graphed in Figure 3. Their overall pattern is much closer to that shown in Figure $2 b$ than the pattern shown in Figure 2a. That is, the line representing contour ratings is approximately straight and parallel to the $\mathrm{X}$ axis, showing that subjects perceived strong contour throughout the sequence of displays. Nevertheless, there is a slight dip in the center of this line. On investigation, this dip was found to be statistically significant. A Pearson correlation of .89 (significant and the .01 level) was found to exist between the mean normalized brightness ratings and subjective contour ratings. The relationship between the two was calculated to be

$$
\mathrm{C}=|0.17 \mathrm{~B}+3.46|
$$

by the method of least squares, where $C$ represents the mean contour rating and $B$ represents the mean brightness rating. Notice that the gradient is slight compared with the gradient close to one which might

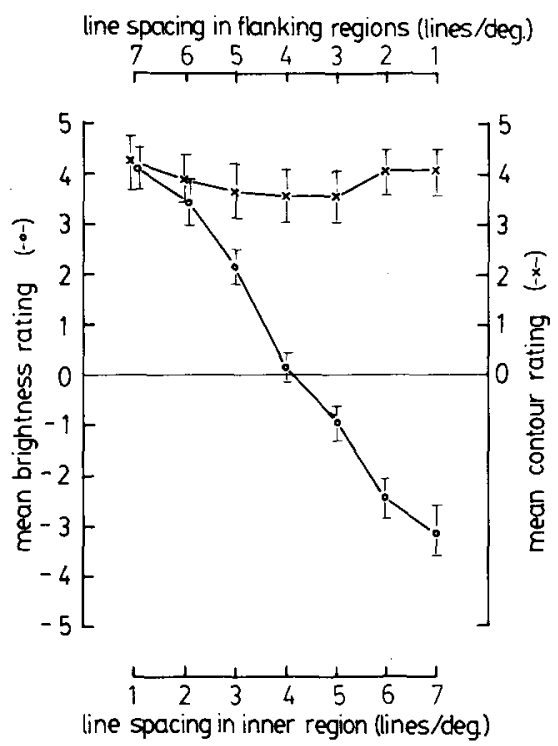

Figure 3. Graph showing mean brightness ratings and mean contour ratings plotted against the line spacing in inner and flanking regions of the display sequence. Vertical bars represent two standard errors on either side of the mean.

be expected were subjective contours perceived brightness gradients. Notice, also, the large constant which indicates that contour is strong in the absence of brightness.

The principle conclusion that may be drawn is that subjective contours are not necessarily brightness gradients. However, the correlation between subjective brightness and subjective contour does suggest that subjective brightness might, to some extent, influence perceived contour. This might be due to some small influence of local brightness effects. Alternatively, gestalt- contingent theories of subjective contours can account for this correlation merely by allowing that a subjective form that differs in brightness from surrounding regions will be more distinct than one that does not.

\section{EXPERIMENT 2}

The second experiment was derived from a study by Kennedy (1978). He showed that the subjective disk was weakened as the radial inducing lines forming a subjective disk (as in Figure 4a) became more tangential to the disk's circumference (as in Figure 4b). $\mathrm{He}$ interpreted this in terms of local effects occurring at the ends of lines; as the inducing lines became more tangential, these small bright regions would be grouped less and less within the region of the disk, the disk brightness would be reduced, and the disk boundary would be correspondingly reduced.

An alternative explanation can be derived from gestalt-contingent theories. Perhaps altering the angle of the inducing lines away from radial positions 
(a)

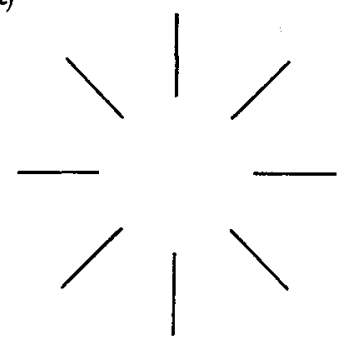

(b)

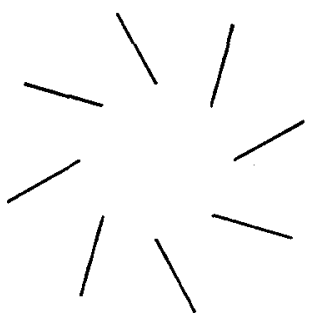

Figure 4. A version of Kennedy's (1978) demonstration with which he showed that perpendicular inducing lines (a) give a stronger subjective disk than do oblique inducing lines (b).

(a)

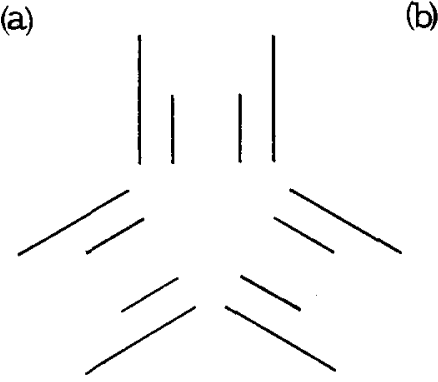

Figure 5. Two flgures which induce subjective triangles. Subjects report a stronger subjective effect in (b), in which inducing lines are oblique to the triangle.

reduces the gestalt for a disk, and, therefore, also the brightness effects. Recently, Parks (1980) used a manipulation involving a subjective triangle to disentangle form perception and brightness. With this pattern, he was able to produce a figure in which the less perpendicular the inducing lines, the greater the impression of stratification in depth between the subjective triangle and surrounding regions. He concluded that this was evidence against the idea that line-end brightness causes subjective contours. However, stratification and contour strength are not necessarily related (Kennedy, 1975), and so the present experiment was designed to use a similar manipulation, involving an induced subjective triangle, and have subjects rate both brightness and contour.

In Figure 5a, the inducing lines are perpendicular to a triangular region; any line-end brightness should be maximally effective within this triangle. In Figure $5 \mathrm{~b}$, the inducing lines can be seen as complete line triangles overlaid by an opaque white triangle, a much stronger gestalt. The inducing lines in this second pattern impinge at $60 \mathrm{deg}$, which should reduce brightness according to local effects theories. Thus, local effects theories predict stronger brightness and contour effects in Figure 5a and gestalt contingent theories predict stronger brightness and contour effects for Figure $5 b$.

\section{Method}

The subjects and the training procedure were the same as those for the previous experiment. The stimuli consisted of patterns as reproduced in Figures 4 and 5 . They were drawn on $81 / 2 \times 11$ in. pieces of illustration board and had the following dimensions. The central disks in the patterns reproduced in Figure 4 were $4 \mathrm{~cm}$ in diameter, and the sides of the subjective triangles in Figure 5 measured $5.5 \mathrm{~cm}$.

\section{Results}

The results are set out in Table 1 . Figure 4a was given a significantly higher rating for both contour and brightness than Figure $4 \mathrm{~b}$, confirming Kennedy's observations. Figure $5 b$ received significantly stronger ratings for both contour and brightness than Figure 5a, supporting the view that gestalt factors, and not local brightness effects, can better account for the stronger effect in Figure 4a.

\section{GENERAL DISCUSSION}

Experiment 1 established that subjective contours are not necessarily brightness gradients. Experiment 2 showed that gestalt factors are more important than the angle of lines impinging in the determination of subjective brightness and contour effects. Both of these findings are incompatible with local effects theories of subjective contours, and both are in accord with the gestalt-contingent view that subjective contours are the edges of subjective forms.

The problem remains as to the exact mechanism whereby a subjective form comes to seem brighter or darker than its surroundings. Kanizsa (1976) suggested that the mechanism is contrast. Thus, the subjective strip formed in Figure 1 will contrast with the flanking strips to seem brighter when the flanks contain a greater density of line elements, and contrast with surrounding regions to seem relatively darker when the flanks contain fewer elements. One problem with this explanation is that thin lines contribute little to a region's overall density, and it is hard to see why they should exert much influence in the form of contrast on adjacent regions. Indeed, it was the efficacy of thin lines in inducing subjective contour and bright-

Table 1

Mean Brightness and Contour Ratings for the Patterns Given in Figures 4 and 5

\begin{tabular}{cccc}
\hline \multicolumn{1}{c}{ Pattern } & Figure & Contour & Brightness \\
\hline Subjective Disk With Perpendicular Inducing Lines & $4 \mathrm{a}$ & $3.1 \dagger$ & $2.0 \dagger$ \\
Subjective Disk With Oblique Inducing Lines & $4 \mathrm{~b}$ & 1.8 & .9 \\
Subjective Triangle With Perpendicular Inducing Lines & $5 \mathrm{a}$ & 1.9 & .8 \\
Subjective Triangle With Oblique Inducing Lines & $5 \mathrm{~b}$ & $2.8 \dagger$ & $1.9^{*}$ \\
\hline
\end{tabular}

Note-Significance levels indicate significantly greater mean ratings for a given figure compared with its comparison figure (two-tailed test). $\quad{ }^{*} p<.05 \quad t p<.01$ 
ness effects that led Frisby and Clatworthy (1975) to first introduce the idea of special line-end contrast mechanisms.

Therefore, it is worth considering an alternative to the contrast explanation. Day and Jory (1978) and Kennedy (1979) have already offered assimilation as a local effects mechanism. However, there is also the possibility of assimilation based on the gestalt. Simply stated, when the visual field is divided into subjectively defined regions, these regions may take on a tint of the color and brightness of inducing elements contained within them. This theory has the advantage of accounting for the efficacy of lines in inducing subjective effects in that thin lines relatively widely spaced are known to be among the most effective inducers of assimilation (Helson, 1963; Helson \& Rowles, 1959).

A second reason for giving assimilation a role in subjective effects is the discovery that color assimilation, and not color contrast, occurs in certain versions of Kanizsa's triangle pattern when the inducing elements are colored (Varin, 1971; Ware, 1980).

Finally, there is some anecdotal evidence which links subjective contours to assimilation effects. Writing about Bezold's (1876) assimilation demonstrations, Evans (1948) noted that "these effects are so strong to most observers that a hazy line is seen vertically through the figure at the ends of the black line part of the figure." Apparently, subjective contours are not incompatible with Bezold assimilation!

\section{REFERENCES}

Bezold, W. von. The theory of colour. Boston: Prang, 1876. Bradley, D. R., \& Dumais, S. T. Ambiguous cognitive contours. Nature, 1975, 257, 582-584.

DAY, R. H., \& JoRy, M. K. Subjective contours, visual acuity, and line contrast. In J. C. Armington, J. Krauskopf, \& B. R. Wooten (Eds.), Visual psychophysics and physiology. New York: Academic Press, 1978.

Evans, R. M. An introduction to colour. New York: Wiley, 1948.

Frisby, J. P., \& Clatworthy, J. L. Illusory contours: Curious cases of simultaneous brightness contrast? Perception, 1975, 4, 349-357.

Ginsburg, A. P. Is the illusory triangle physical or imaginary? Nature, 1975, 257, 219-220.

Gregory, R. L. Vision with isoluminant colour contrast: 1. A projection technique and observations. Perception, 1977. 6, 113-119.
Helson, H. Studies of anomalous contrast and assimilation. Journal of the Optical Society of America, 1963, 53, 179-184.

Helson, H., \& Rowles, F. H. A quantitative study of the reversal of classical lightness contrast. American Journal of Psychology, 1959, 72, 530-538.

JoRY, M. K., \& DAY, R. H. The relationship between brightness contrast and illusory contours. Perception, 1979, 8, 3-9.

Kanizsa, G. Margini quasi-percettivi in campi con stimolazione omogenea. Rivista di Psicologia, 1955, 49, 7-30.

Kanizsa, G. Some new demonstrations of the role of structural factors in brightness contrast. In v. S. Ertel, L. Kemmler, \& M. Stadler (Eds.), Gestalt Theorie in der modernen Psychologie, 1975.

Kanizsa, G. Subjective contours. Scientific American, 1976, 234(4), 48-64.

KenNedy, J. M. Depth at an edge, coplanarity, slant depth, change in direction and change in brightness in the production of subjective contours. Italian Journal of Psychology, 1975, 2, 107-123.

Kennedy, J. M. Illusory contours and the ends of lines, Perception, 1978, 7, 605-607.

KENNEDY, J. M. Subjective contours, contrast and assimilation. In C. F. Nodine \& D. F. Fisher (Eds.), Perception and pictorial representation. New York: Praeger, 1979.

Kennedy, J. M., \& LeE, H. A figure-density hypothesis and illusory contour brightness. Perception, 1976, 5, 387-392.

Parks, T. E. The subjective brightness of illusory figures: Is stratification a factor? Perception, 1980, 9, 361-363.

VARIN, D. Fenomeni di contrasto e diffusione chromatica nell' organizzazione spaziale del campi percettivo. Rivista di Psicologia, 1971, 65, 101-128.

Ware, C. Coloured illusory triangles due to assimilation. Perception, 1980, 9, 103-107.

\section{NOTES}

1. There is a third kind of theory which stands outside this dichotomy. This is Ginsburg's (1975) theory that at some level in the visual system subjective forms (and hence their contour boundaries) are present because of spatial Fourier filtering of the display. In this theory, form and brightness are present in one operation. The present studies do not address this third alternative.

2. This refers to a display with black pattern elements on a white ground. In a display with white elements on a black ground, the effect will be perceived as a darkening.

3. The two experiments reported in this paper were part of a larger study in which subjects were required to rate 60 different patterns for contour and brightness. The display sequence reported here was given a block within other blocks of displays. There was no evidence that prior exposure to other patterns used in the study influenced mean ratings.

(Received for publication February 28, 1980; revision accepted March 6, 1981.) 